\title{
Características de la glicerina obtenida del proceso de la reacción del metóxido de sodio en la producción del Biodiesel
}

\section{Characteristics of glycerin obtained from the reaction process of sodium methoxide in the production of Biodiesel}

\author{
TORRES-RIVERO, Ligia Adelayda $\dagger^{*}$, BEN-YOUSSEF, Brants Cheriff y PÉREZ-GASCA, María \\ Fernanda
}

Instituto Tecnologico de Cancun

ID $1^{\text {er }}$ Autor: Ligia Adelayda, Torres-Rivero / ORC ID: 0000-0002-3303-3437

ID $1^{\text {er }}$ Coautor: Brants Cheriff, Ben-Youssef / ORC ID: 000-0003-1468-5365

ID $2^{\text {do }}$ Coautor: María Fernanda, Pérez-Gasca

\begin{abstract}
Resumen
El biodiesel constituye una de las fuentes alternas de energía obtenido de los aceites comestibles usados de los diferentes tipos de cocinas como un recurso renovable. El subproducto derivado del proceso de la reacción la transesterificación en la elaboración de biodiesel es la glicerina. La solución acuosa de glicerina se filtra para eliminar las impurezas y luego se neutraliza con $\mathrm{NaOH} 1 \mathrm{~N}$. El objetivo del presente trabajo fue identificar las características de esta glicerina y sus aplicaciones y su futuro uso, la caracterización realizada a este subproducto del cual se recolecto 4 litros en botellas ámbar, el rango $\mathrm{pH}$ entre 6 y 7 , densidad $1.24 \mathrm{~g} / 1$, viscosidad .897 pascal seg., es posible recuperar el metanol a una pureza de $58 \%$ en peso, la glicerina se utiliza en muchos productos de consumo, posee una baja toxicidad sus propiedades son estabilidad y compatibilidad hacia otros compuestos químicos. Las aplicaciones de este subproducto son la elaboración de jabón líquido, desengrasante, limpieza de pisos, es un compuesto no irritante, biodegradable y reciclable
\end{abstract}

\section{Glicerina, Uso, Biodiesel}

\begin{abstract}
Biodiesel is one of the alternative sources of energy obtained from the edible oils used in different types of kitchens as a renewable resource. The by-product derived from the reaction process transesterification in the production of biodiesel is glycerin the aqueous glycerin solution is filtered to remove the impurities and then neutralized with $1 \mathrm{~N} \mathrm{NaOH}$. The objective of this work was to identify the characteristics of this glycerin and its applications and future use, the characterization made of this by-product from which it was collected. liters in amber bottles, the $\mathrm{pH}$ range between 6 and 7, density $1.24 \mathrm{~g} / 1$, viscosity .897 pascal sec., it is possible to recover methanol to a purity of $58 \%$ by weight, glycerin is used in many products of consumption, has a low toxicity, its properties are stability and compatibility towards other chemical compounds. The applications of this by-product are the manufacture of liquid soap, degreaser, floor cleaning, it is a non-irritating, biodegradable and recyclable compound.
\end{abstract}

Gliceryn, Use, Biodiesel

Citación: TORRES-RIVERO, Ligia Adelayda, BEN-YOUSSEF, Brants Cheriff y PÉREZ-GASCA, María Fernanda. Características de la glicerina obtenida del proceso de la reacción del metóxido de sodio en la producción del Biodiesel. Revista de Energía Química y Física. 2019. 6-18: 18-28

\footnotetext{
* Correspondencia al Autor (Correo electrónico: torlia@ hotmail.com)

$\dagger$ Investigador contribuyendo como primer Autor
} 


\section{Introducción}

Los aceites residuales como materia prima para obtención de biodiesel tiene unas ventajas amigables con el medio ambiente, evita la disposición en los drenajes de las cocinas económicas, y de nuestra propia casa del cual se produce un combustible limpio, el cual se lleva a cabo mediante la reacción de transesterificación. Sometiendo el aceite residual a un tratamiento que incluye deshidratación y filtración en primera medida, para luego pasar a los procesos de esterificación (catálisis ácida $\mathrm{H}_{2} \mathrm{SO}_{4}$ ) y transesterificación (alcalina $\mathrm{NaOH}$ ) con el fin de mejorar el rendimiento de la obtención de biodiesel a partir de aceites residuales y de glicerina (Diguilio E, et all 2015)

La fuente de obtención glicerina es por la producción de biodiesel, la separación del glicerol obtenido a partir del biodiesel es relativamente simple, se requiere de un lavado para eliminar el metanol y los ácidos grasos y jabones que pueda arrastrar.

La fórmula de la glicerina está compuesta por 1, 2,3 propanotriol, 1, 2,3 trihidroxipropano, glicerol o glicerina $\left(\mathrm{C}_{3} \mathrm{H}_{8} \mathrm{O}_{3}\right)$ es un alcohol con tres grupos hidroxilos $(-\mathrm{OH})$, por lo que podemos representar la molécula como se indica en la siguiente figura 1 :

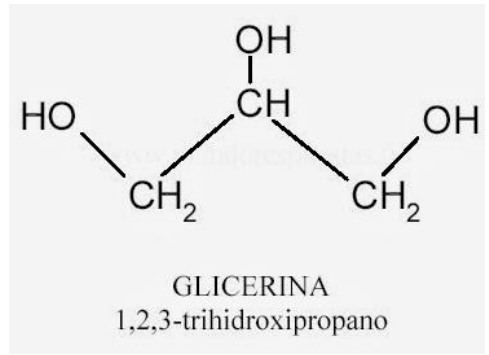

Figura 1 Molecula de glicerina, fuente

Sus características físicas son líquido incoloro, inodoro, higroscópico y de sabor dulce. Las propiedades físicas del glicerol, como punto de ebullición elevado $\left(290{ }^{\circ} \mathrm{C}\right)$, punto de fusión bajo $\left(18{ }^{\circ} \mathrm{C}\right)$, viscosidad, densidad, etc. pueden ser explicadas por su extendido enlace intermolecular de hidrógeno. El glicerol atrae y capta la humedad del ambiente, los tres grupos $\mathrm{OH}$ que posee son responsables de su solubilidad en agua y alcohol, resultando insoluble en hidrocarburos.
La glicerina se caracteriza por ser un compuesto muy estable y compatible con muchas sustancias. Así mismo, presenta propiedades físicas y químicas que permiten su utilización y aplicación en innumerables procesos químicos, principalmente para la industria farmacéutica, cosmética y alimentaria. El biodiesel se obtiene a partir de aceites vegetales, el método más habitual es la transformación de estos aceites vegetales a través de un proceso de combinación con alcohol metílico e hidróxido de sodio $(\mathrm{NaOH})$, conocido como reacción de transesterificación, obteniéndose glicerina como subproducto.

La cantidad de aceite y alcohol que se emplean se determinan por relaciones estequiométricas. En el caso del hidróxido de sodio cuando se trabaja con el aceite de cocina usado se requiere usar más lejía que con el aceite nuevo, no para catalizar la reacción, sino para neutralizar los ácidos grasos libres que se forman en el aceite al utilizarlo en el proceso de freído. Cuanto más se haya calentado y más tiempo haya estado caliente, más ácidos grasos libres contendrá, y hará falta más hidróxido de sodio para neutralizarlos

La glicerina que se obtiene de la producción de la producción de biodiesel presenta una concentración máxima del $60 \%$ de glicerina, carece de valor, ya que contiene gran cantidad de jabones, catalizador alcalino y metanol y este compuesto es ambientalmente peligrosa;, para poder aprovecharla. Los biocombustibles reducen las emisiones de gases de efecto invernadero. El uso de los biocombustibles genera una menor contaminación ambiental y es considerada una alternativa viable al agotamiento ya sensible de energías fósiles, como lo son el gas y el petróleo.

Las características físico-químicas de la glicerina cruda han sido ampliamente descritas en la bibliografía internacional. Algunos autores las refieren de acuerdo al tipo de reacción que caracteriza su obtención (transesterificación, saponificación e hidrólisis) (Betancourt-Aguilar et all 2016). A pesar que el proceso de transesterificación es el que produce los porcentajes más bajos de glicerina y más altos de las impurezas es el que tiene una mayor aplicación. 
La transesterificación catalizada por base (usando metanol como alcohol y $\mathrm{NaOH}$ como el catalizador) convierte las grasas y aceites en los esteres de metilo de los tres ácidos grasos individuales Con la producción de biodiesel por el proceso de la transesterificación del aceite vegetal reusado con metanol en medio de la catálisis acida y básica, la producción de glicerina, se obtiene como subproducto de este proceso. La glicerina es gelatinosa y más oscura en el fondo y los metilésteres (biodiésel) quedan encima de la mezcla. De esta forma glicerina se mantiene semilíquida (solidifica por debajo de $38^{\circ} \mathrm{C}$ ). (Véase figura 1 )
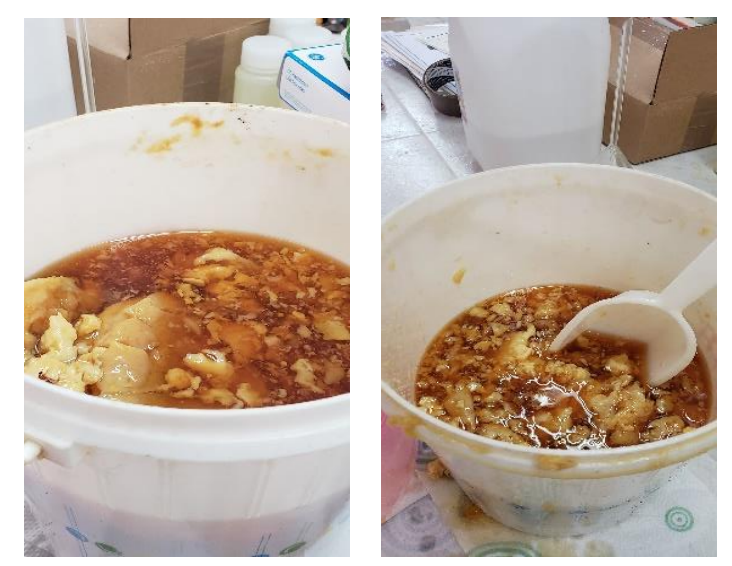

Figura1 Contenedor de glicerina sólida Fuente: Elaboración Propia

Se separa la glicerina y el biodiésel por un medio de un embudo de separación. La glicerina semilíquida es de color marrón oscuro; el biodiésel es del color de la miel. Se separa la glicerina y cuando empiece a salir biodiésel se cierra la llave del embudo de decantación. Si cae algo de biodiésel en el matraz Erlenmeyer de la glicerina es fácil recuperarlo cuando la glicerina se espesa. (Véase figura 2)

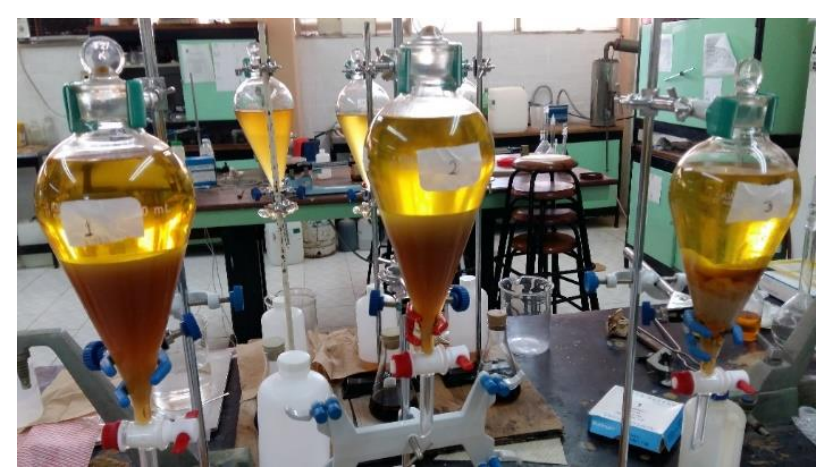

Figura 2 Separación de la glicerina del biodiesel Fuente: Elaboración Propia

\section{Características de la glicerina}

En la siguiente Tabla 1 se muestra las especificaciones de calidad para cada tipo de glicerina. Se observan las especificaciones de calidad y los límites normales para los contaminantes presentes, cuyos valores sirven para comparar las concentraciones de los compuestos comunes entre los tres tipos de glicerinas. La glicerina cruda es el principal subproducto que se obtiene durante el proceso de producción de biodiesel, por lo que su composición química está contaminada con significativas cantidades de metanol, agua, jabones y sales.

\begin{tabular}{|c|c|c|c|}
\hline Propiedades & Glicerina cruda & $\begin{array}{l}\text { Glicerina grado } \\
\text { técnico }\end{array}$ & $\begin{array}{l}\text { Glicerina refinado } \\
\text { grado USP }(99,7 \%)\end{array}$ \\
\hline Contenido de glicerol & $40 \%-88 \%$ & $98 \% \min$. & $99,7 \%$ \\
\hline Ceniza & $2 \%$ máx. & NA & $\mathrm{NA}$ \\
\hline Contenido de humedad & $\mathrm{NA}$ & $2 \%$ máx. & $0,3 \%$ máx. \\
\hline Cloruros & $\mathrm{NA}$ & $10 \mathrm{ppm}$ máx. & 10 ppm máx. \\
\hline Color & NA & 40 máx. $\left(\mathrm{Pr}-\mathrm{Co}_{0}\right)$ & 10 máx. (APHA) \\
\hline Gravedad especifica & $\mathrm{NA}$ & 1,262@25으 & 1,2612 min. \\
\hline Sulfato & $\mathrm{NA}$ & NA & $20 \mathrm{ppm} \mathrm{Max}$ \\
\hline Análisis & $\mathrm{NA}$ & $\mathrm{NA}$ & $\begin{array}{l}99 \%-101 \% \text { (base } \\
\text { seca) }\end{array}$ \\
\hline Metales pesados & $\mathrm{NA}$ & 5 ppm máx. & 5 ppm máx. \\
\hline Componentes clorados & $\mathrm{NA}$ & $30 \mathrm{ppm}$ máx. & $30 \mathrm{ppm}$ máx. \\
\hline Residuos de ignicion & $\mathrm{NA}$ & $\mathrm{NA}$ & $100 \mathrm{ppm}$ máx. \\
\hline Ácidos grasos y esteres & NA & 1 máx. & 1.000 máx. \\
\hline Agua & $12 \%$ maxx. & $5 \%$ máx. & $0,5 \%$ máx. \\
\hline $\mathrm{pH}$ (solución 10\%) & $4-9$ & $4-9,1$ & $\mathrm{NA}$ \\
\hline Residuos orgánicos & $2 \%$ máx. & $2 \%$ máx. & $\mathrm{NA}$ \\
\hline
\end{tabular}

Tabla 1 Especificaciones de calidad para cada tipo de glicerina

\section{Legislación}

El glicerol se aborda con la especificación "glicerina libre" (los términos "glicerina" y "glicerol" frecuentemente se usan como sinónimos) mientras que los mono-, di- y triacilgliceroles están limitados en forma combinada por la especificación "glicerol total" que también incluye el valor para "glicerol libre" punto de inflamación, aunque la norma ASTM D6751 ahora contiene una especificación separada para control de alcohol

La norma europea en 14214 contiene especificaciones similares, aunque en este caso los tres tipos de acilgliceroles están sujetos a límites individuales. Estos límites individuales, junto con la especificación de "glicerina libre" se pueden combinar para obtener aproximadamente la especificación de glicerol total en 14214. 
Las propiedades que se indican en estas especificaciones incluyen el poder calorífico, número de cetano, densidad, viscosidad, contenido de ceniza, corrosión al cobre, contenido de agua, azufre, glicerina, entre otras (ASTM International, 2015; CEN, 2014)

En México se han impulsado políticas para fomentar los biocombustibles, uno de los primeros esfuerzos para impulsar el uso de energías limpias son la Ley de Promoción y Desarrollo de los Bioenergéticos (LPDB, 2008) y la Ley para el Aprovechamiento de Energías Renovables y el Financiamiento de la Transición Energética (LAERFTE, 2013)

\section{Tipos de glicerina}

Glicerina cruda: Es el producto contenido en la corriente de salida del proceso de transesterificación y contiene una gran cantidad de metanol, agua, jabones y sales. Normalmente tiene un contenido de glicerol entre 40 y $88 \%$ en peso. Glicerina grado técnico: Es un producto de alta pureza con la mayoría de sus contaminantes completamente removidos. La concentración no debe ser inferior al 98\%

Glicerina USP: Con una concentración del $99,7 \%$ es la que cumple con la norma USP (United States Pharmacopeia) y el Food Chemicals Codex (FCC) y por lo tanto es apta para uso alimenticio, farmacéutico y cosmético.

\section{Impacto ambiental}

Glicerina la contaminación por compuestos orgánicos volcados en ríos y arroyos es la disminución de oxígeno disuelto en el agua como consecuencia de su consumo para los procesos de degradación biológica de dichos compuestos. Esta disminución de oxígeno puede ocasionando un proceso de degradación de las especies de plantas, animales y microorganismos en dicho medio impidiendo el paso de la luz para que se lleve a cabo el proceso de la fotosíntesis. Se debe recordar que la glicerina cruda contiene jabón (saponificación de los ácidos grasos con el hidróxido de sodio agregado en exceso para obtener una transesterificación completa), catalizador, sales y metanol.

El metanol contenido en la glicerina convierte a ésta en un residuo peligroso, dado que este producto es sumamente tóxico.
La dosis letal del metanol está estimada en 30-240 ml (20-150 gramos), la dosis tóxica mínima es aproximadamente de $100 \mathrm{mg} / \mathrm{kg}$. (Ávila Orozco Francisco David 2015)

\section{Principales usos del glicerol.}

La elaboración de cosméticos, jabones de tocador. La glicerina en el área de la medicina se utiliza en la elaboración de medicamentos en forma de jarabes, Como baño calefactor para temperaturas superiores a los $250{ }^{\circ} \mathrm{C}$; Lubricación de maquinarias específicas. Producción de alimentos y medicamentos (por no ser tóxica), de petróleo, etc.; En disciplinas militares para la fabricación de explosivos, como la nitroglicerina así como para enfriar los cañones de las armas de fuego.

\section{Descripción del método}

La generación de grandes cantidades de aceites comestibles usados, nos pone en alerta la falta de conciencia, el desconocimiento en la reutilización del aceite usado comestible, como la falta de un correcto reciclaje nos lleva a un grave problema ambiental y de salud pública. Los métodos analíticos que se emplearon para cuantificar el proceso de caracterización de la glicerina obtenida del proceso de transesterificación de la producción del biodiésel son el siguiente:

Siguiendo la Metodología de acuerdo a la legislación UNE EN14214. Siguiendo los procedimientos estándar de empresas COPEC para los análisis de combustibles de acuerdo con ASTM Standard Methods (ASTM 2015).

Se analizaron las características composicionales y fisico de la glicerina obtenida, tales como densidad, viscosidad, temperatura de ebullición, $\mathrm{pH}$, recuperación del metanol.

En cuanto a las muestras se cuenta con 4 litros de glicerina cruda obtenida del proceso del biodiesel de diferentes tipos de aceites recolectados del centro de acopio de aceite comestible usado ubicado en el Instituto Tecnológico de Cancún, la cual se obtuvo 8 tipos de muestras. 


\section{Análisis físico del aceite}

A las muestras de glicerina se realizaron análisis para su identificación física, utilizando las normas que podríamos usar para cada determinación y adoptar la que esté acorde a nuestras condiciones:

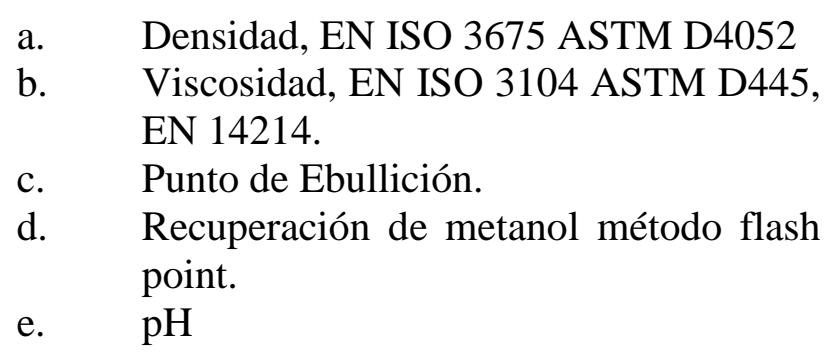

\section{Diseño de Experimentos}

El diseño de experimentos seleccionado es de 2 niveles y 3 variables como se muestra a continuación: con el STATGRAPHICS Centurión XVIII,

El diseño de experimentos seleccionado es un diseño factorial $2^{3}$, tres factores y dos niveles (Representados con signos “- $\mathrm{y}+$ ” para los niveles menor y mayor).

\section{Viscosidad cinemática}

La prueba fue realizada como indica la metodología (pruebas de laboratorio realizada la glicerina obtenida del biodiesel relación molar 6:1. Esta prueba fue llevada a cabo viscosímetros capilares Ostwald y Cannon. Debido al tamaño pequeño del baño maría se implementó un montaje de equipo para la realización del método. (Véase figura 3).
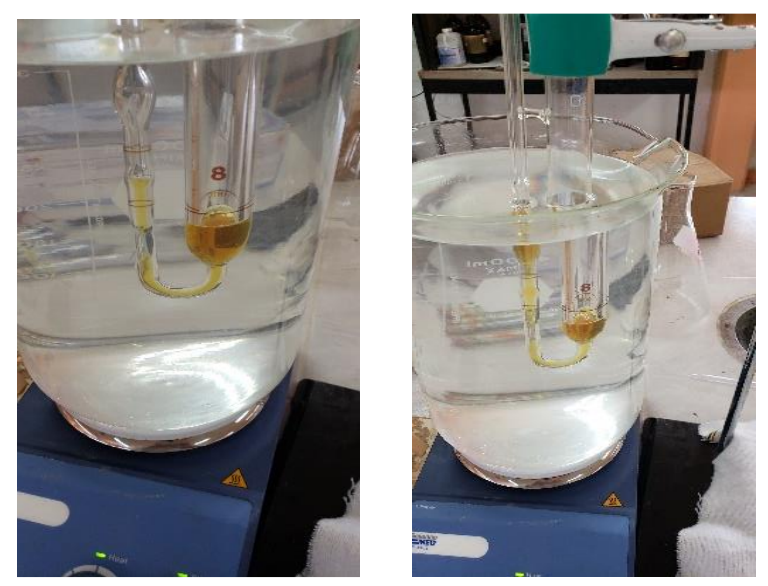

Figura 3 Determinaciones de la viscosidad de la glicerina Fuente: Elaboración Propia
Viscosidad cinemática a 40 grados centígrados $\mathrm{El}$ método de ensayo prestablecido bajo esta norma se basa en ASTM D445 Kinematic Viscosity of Transparent and Opaque Liquids, the Calculation of Dynamic Viscosity, su unidad de medida serán $\mathrm{mm} 2 / \mathrm{s}$, con un rango de aceptación 3,5 - 5. Este método de prueba específica un procedimiento para la determinación de la viscosidad cinemática, del petróleo líquido y sus productos, tanto transparentes como opacos, midiendo el tiempo para que un volumen de líquido fluya bajo la influencia de la gravedad a través de un viscosímetro capilar de vidrio calibrado.

\section{Densidad a 15 grados centígrados}

La prueba fue realizada como indica la metodología (pruebas de laboratorio realizadas a la glicerina, con la relación molar 6:1 del proceso de esterificación para obtener biodiesel. Ya sea por la concentración de catalizadores, tiempo de agitación o temperatura. Se basó Norma EN14214.

Los valores se miden en un hidrómetro a la temperatura de referencia o a otra temperatura conveniente, y las lecturas se corrigen a la temperatura de referencia; los valores obtenidos a una temperatura distinta a la temperatura de referencia son las lecturas del hidrómetro y las mediciones que no corresponden a la densidad. $\mathrm{Su}$ método de ensayo consiste en llevar la muestra a una temperatura específica y una porción de la muestra transferirla a una probeta, cilindro de hidrómetro que se ha llevado a aproximadamente la misma temperatura. (Véase figura 4) se coloca la glicerina en la probeta el hidrómetro y su contenido se colocan en un baño de temperatura constante para evitar una variación excesiva de temperatura durante la prueba.
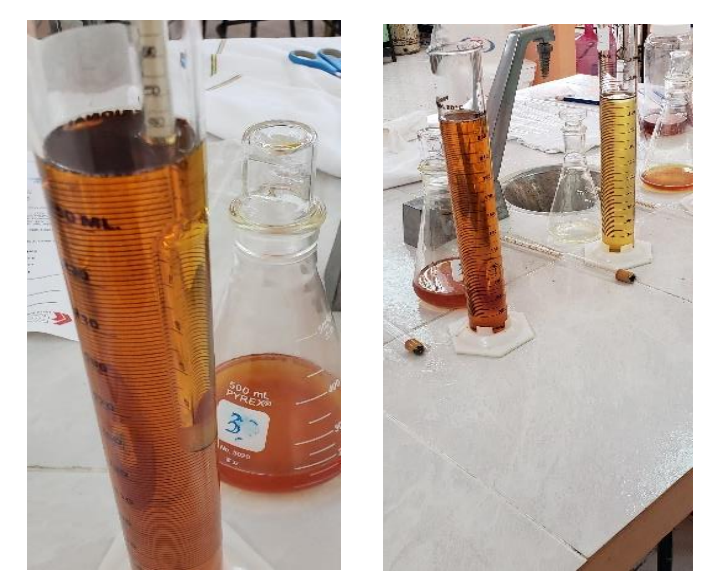

Figura 4 Determinación de la densidad Fuente: Elaboración Propia

TORRES-RIVERO, Ligia Adelayda, BEN-YOUSSEF, Brants Cheriff y PÉREZ-GASCA, María Fernanda. Características de la glicerina obtenida del proceso de la reacción del metóxido de sodio en la producción del Biodiesel. Revista de Energía Química y Física. 2019. 


\section{pH}

Metodología para este parámetro no tiene una técnica específica, se puede hacer de forma directa o de medición por tira reactiva de $\mathrm{pH}$. La medición de ambas se realizó directamente del vaso de precipitado y por medio de tiras.
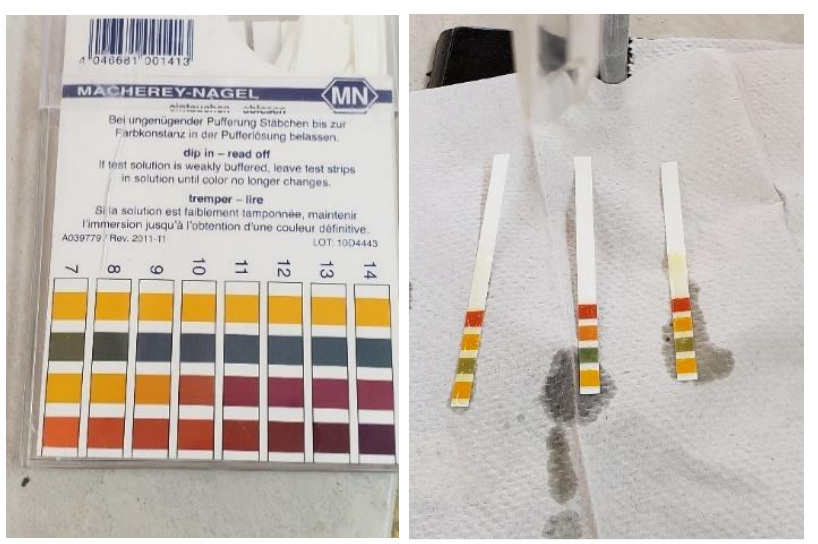

Figura 6 Determinaciones del $\mathrm{pH}$ por tiras reactivas Fuente: Elaboración Propia

\section{Recuperación de Metanol}

Técnica de Roto vapor para recuperación de metanol, y destilación simple

La técnica a utilizar, si se requiere una mayor pureza en la glicerina, es la destilación a vacío (destilación molecular) tras la cual el grado de purificación llega al grado técnico, con un porcentaje en glicerol alrededor del $98 \%$. Sin embargo, siendo este método el más comúnmente utilizado y el que mayor rendimiento posee, es un proceso de alto consumo energético debido a su alta demanda de suministro energético. (Montoro Garcìa Francisco Javier 2016)

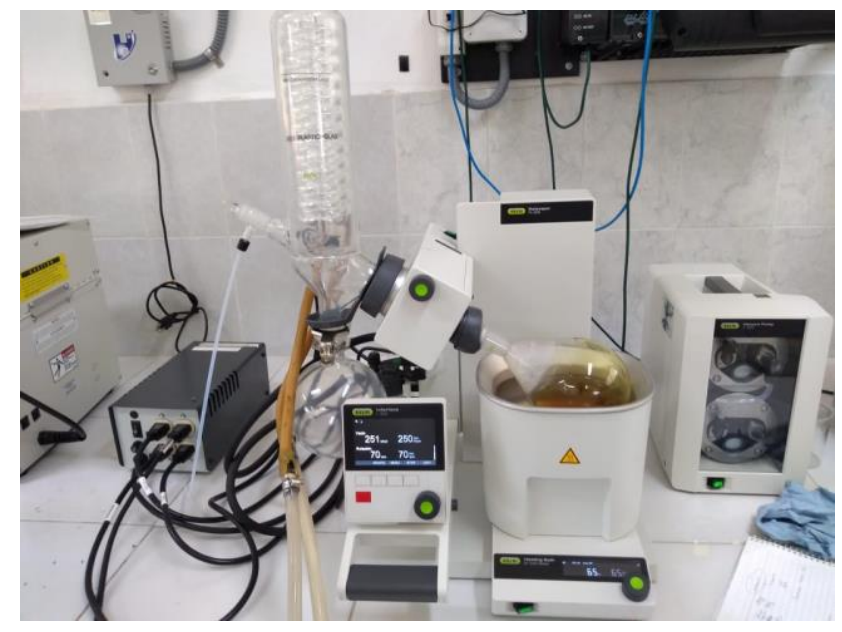

Figura 5 Recuperaciones del metanol roto vapor Fuente: Elaboración Propia
Destilación simple recuperación del methanol
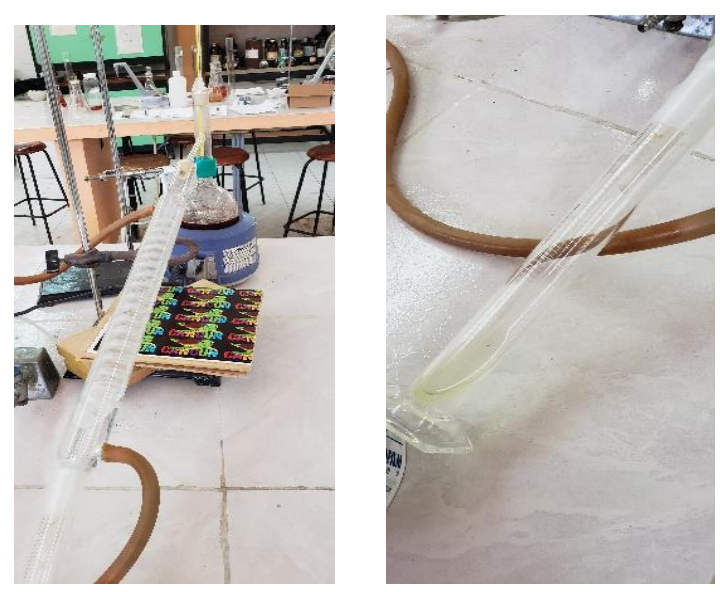

Figura 7 Recuperación de Metanol por destilación Fuente: Elaboración Propia

\section{Punto de Ebullición}

El procedimiento experimental empleado para la determinación de este parámetro es el siguiente: se tomó un tubo Thiele y se llenó con aceite hasta completar $3 / 4$ de su volumen aproximadamente, en un tubo Pasteur se colocó un termómetro, en la siguiente pipeta Pasteur se agregaron $2 \mathrm{ml} \mathrm{de}$ la glicerina a analizar, El procedimiento experimental empleado para la determinación de este parámetro es el siguiente como se indica el figura 8: el cual había sido previamente sellado por uno de sus extremos, se sumergió en el líquido de manera que el extremo abierto quedara en contacto con el mismo, se procedió a fijar el termómetro con la pipeta Pasteur a un soporte universal de manera que estos quedaran dentro del aceite del tubo thiele y se procedió a calentar este haciendo uso de un mechero, cuando se obtuvo un burbujeo constante de la muestra se registró esta temperatura como la temperatura $1 \mathrm{y}$ en el punto en que el líquido comenzó a subir por el capilar se registró la temperatura 2 y luego se realizó un promedio con estos valores y se obtuvo el punto de ebullición de la muestra.

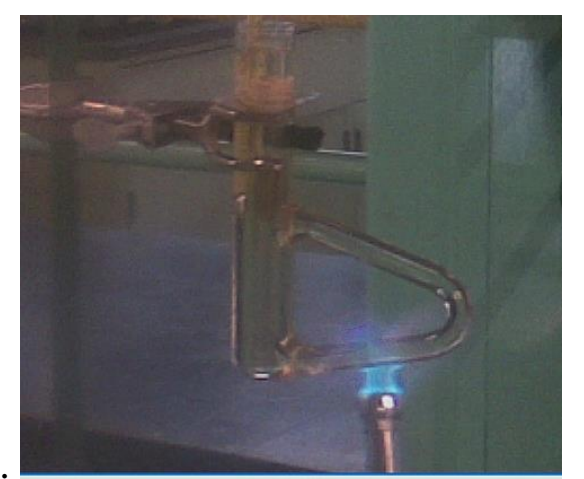

Figura 8 Determinacion del punto de ebullicion de la glicerina

Fuente: Elaboaración Propia

TORRES-RIVERO, Ligia Adelayda, BEN-YOUSSEF, Brants Cheriff y PÉREZ-GASCA, María Fernanda. Características de la glicerina obtenida del proceso de la reacción del metóxido de sodio en la producción del Biodiesel. Revista de Energía Química y Física. 2019. 
Pto de ebullición $=(\mathrm{T} 1+\mathrm{T} 2) \div 2$

Donde:

T1 =Temperatura a la cual se puede observar un burbujeo constante.

T2 =Temperatura a la cual el líquido penetra por el capilar.

Punto de Ebullición = Punto de ebullición de la muestra

\section{Resultados}

\section{Análisis de resultados}

Teniendo ya los resultados de las propiedades físico evaluado, se procedió a realizar un análisis comparativo entre estos valores y los valores referenciales para la glicerina aportada por la revisión de distinta fuentes bibliográficas.

Datos teóricos: $\left(25^{\circ} \mathrm{C}\right)$,

Punto de ebullición $=290{ }^{\circ} \mathrm{C}$

Densidad $=1,26 \mathrm{~g} \backslash \mathrm{mL}$

$\mathrm{pH}=6,5-7,5$

Datos obtenidos experimentalmente: $\left(25^{\circ} \mathrm{C}\right)$

Punto de ebullición $=120.5^{\circ} \mathrm{C}$

Densidad $=1,24 \mathrm{~g} \backslash \mathrm{mL}$

$\mathrm{pH}=7-8$

Recuperación del metanol el 58\%

Viscosidad .894 pas.s

Como se observa al comparar los resultados existe una pequeña variación en los valores obtenidos de forma experimental con respecto a los datos teóricos, pero esto puede estar originado al hecho de que hayan quedado trazas de impurezas en la glicerina. Al ser estas desviaciones tan bajas pudiera asegurarse que dicha glicerina puede ser utilizada sin ningún tipo de riesgos, en la formulación del detergente líquido o para usos cosméticos, elaboración de velas para este caso en particular hay que purificar la glicerina la eliminar el olor que le que a grasas.. Este sería otro trabajo a realizar.

Se recolectaron para este estudio 6 tipos diferentes de aceites del centro de acopio del ITCancún (véase figura 9).
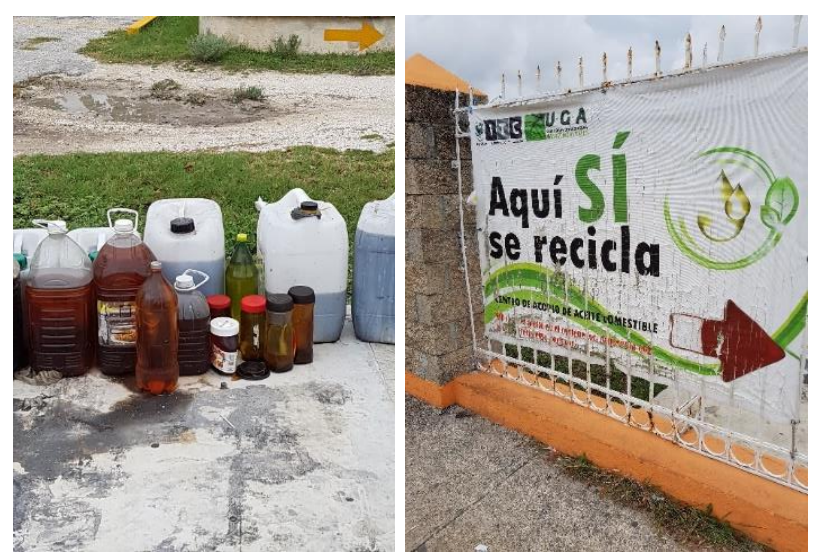

Figura 9 Centro de acopio de ITCancùn Fuente: Elaboración Propia

Se procedió hacer un análisis físico de las condiciones de los aceite, características organolépticas, midiendo $\mathrm{pH}$ y temperatura. Posteriormente se midió el volumen de cada muestra, se filtró para eliminar los residuos del contenido de las muestras, se dejó decantar por 12 horas aproximadamente. Se tomó una muestra de $400 \mathrm{ml}$ de los aceites para determinar índice de acidez, índice de peróxido, humedad, índice de yodo. Se llevó a cabo todo el desarrollo experimental desde la esterificación con catalizador acido, hasta el proceso de transesterificación con el metóxido de sodio para obtener el biodiesel así como la glicerina como se muestra en la siguiente figura No. 10
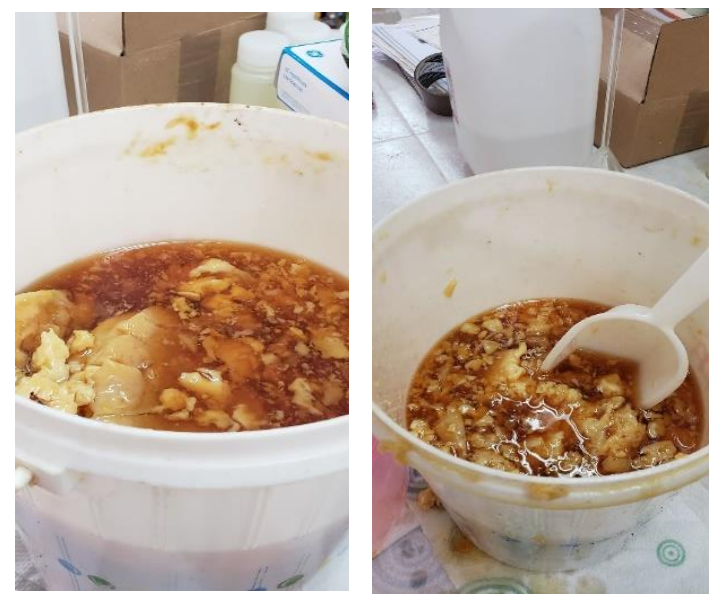

Figura 10 Muestra de glicerina Fuente: Elaboración Propia

De la cual se obtuvieron un total de 4 litros de los diferentes tipos de aceites, se utilizaron las técnicas para biodiesel y algunas de las normas vigentes Europeas o ASTM de EU, ya que en México no se cuenta con normas y legislación para la glicerina como subproducto de biodiesel. 


\section{Punto de Ebullición de la glicerina}

Se obtuvo el siguiente resultado temperatura, y el tiempo que tardaba en ebullir la glicerina contenida en la pipeta Pasteur, dentro del tubo Thiele, como se indica en la tabla no. 2 ,

\begin{tabular}{|r|r|r|}
\hline Tiempo min & Muestra & Temeperaturas \\
\hline 6 & 4 & 70.0 \\
\hline 7 & 2 & 95.0 \\
\hline 10 & 3 & 107.333 \\
\hline 11 & 1 & 95.0 \\
\hline 12 & 5 & 120.0 \\
\hline 15 & 6, & 95.0 \\
\hline
\end{tabular}

Tabla 2 Resultados punto de ebullición de la glicerina Fuente: Elaboración Propia

El tratamiento estadisco se realizó con el software STATGRAPHICS Centurión XVIII Se estimaron 8 muestras las cuales presentaron puntos de ebullición menores a los que reporta la bibliografía.

\section{Variable dependiente: Temperatura C (Parametro Punto de Ebullicion)}

Este procedimiento ejecuta un análisis de varianza de un factor para Temperatura $\mathrm{C}$. Construye varias pruebas y gráficas para comparar los valores medios de Temperatura $\mathrm{C}$ para los 6 diferentes niveles de Tiempo min. Lo que nos arroja que tiene una deviación estandart 15,8919, coeficiente de variación de 15.918 , lo que nos indica que el sesgo estandarizado 0.67799. Este procedimiento construye un gráfico de caja y bigotes para comparar las 6 muestras de Temperatura C. Para un análisis estadístico detallado de estos datos, se tiene el siguiente grafico 1 , que la muestra 5 es la que tiene un punto de ebullición más alto.

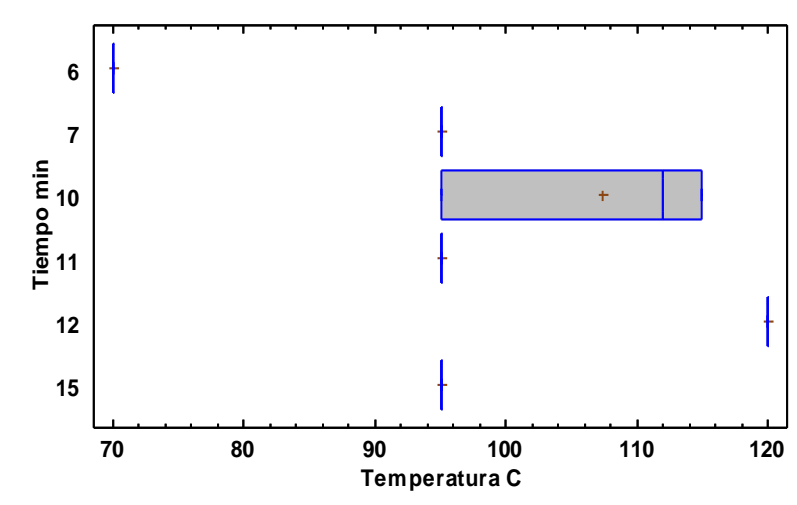

Grafica 1 Punto de ebullición Fuente: Elaboración Propia

\section{Densidad}

Variable dependiente: Muestra

Variable independiente: Densidad (EN-14214)

Este procedimiento construye un gráfico de caja y bigotes para comparar las 7 muestras. De acuerdo a los siguientes resultados, que se muestran en la tabla 3 y grafico 2 , en el cual solo un punto cae dentro de los límites establecidos para la glicerina que es de $1.24 \mathrm{~g} / \mathrm{ml}$.

\begin{tabular}{|r|r|}
\hline \multicolumn{1}{|c|}{ Muestra } & Densidad \\
\hline 1 & .2 \\
\hline 2 & .9 \\
\hline 3 & 1.90 \\
\hline 4 & 1.24 \\
\hline 5 & .39 \\
\hline 5 & .48 \\
\hline 7 & .52 \\
\hline 8 & .24 \\
\hline
\end{tabular}

Tabla 3 Resultados obtenidos de la densidad Fuente: Elaboración Propia

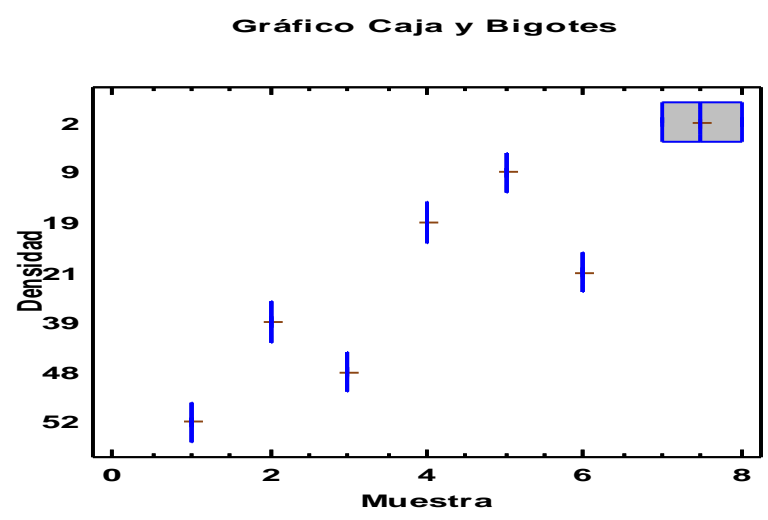

Grafico 2 Densidad

Fuente: Elaboración Propia

La densidad es uno de los parámetros más importantes de los combustibles y biocombustibles, ya que los sistemas de inyección, bombas e inyectores deben suministrar la cantidad de combustible con precisión para proporcionar la combustión adecuada. La densidad del biodiesel depende del contenido en ésteres y de la cantidad remanente de alcohol, por lo tanto, esta propiedad viene determinada fundamentalmente por el aceite vegetal elegido $\mathrm{y}$, en cierta medida, por las etapas de purificación aplicadas. Esta tabla muestra intervalos de confianza del $95.0 \%$ para los coeficientes en el modelo. Los intervalos de confianza muestran con qué precisión pueden estimarse los coeficientes dados la cantidad de datos disponibles, y el nivel de ruido que está presente. 
Gráfica del Modelo Ajustado

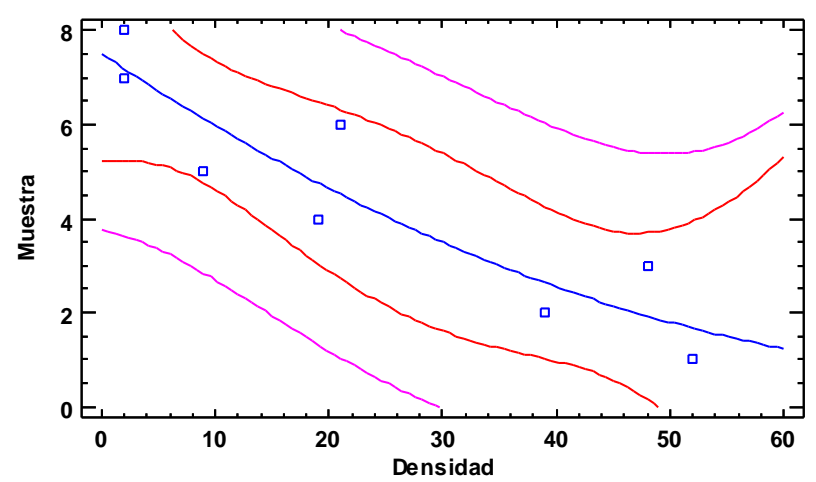

Viscosidad

La viscosidad tanto de los aceites como del biodiesel se determinó con la ayuda de un viscosímetro Cannon. Fue necesario retirar previamente el exceso de disolvente a las muestras. Se utilizaron $50 \mathrm{~mL}$ de muestra previamente calentada a $40 \mathrm{oC}$

A partir del tiempo de flujo, $t$, expresado en segundos, se obtiene la viscosidad cinemática expresada en centistokes, $v=C \cdot t$, siendo $C$, la constante de calibración del sistema de medida en cSt.s, que viene dada por el fabricante del aparato, siendo en este caso $0.04058 \mathrm{~mm} 2 / \mathrm{s} \mathrm{a} 40$ ${ }^{\circ} \mathrm{C}$ y t el tiempo de flujo en segundos.

Este procedimiento genera un diagrama de dispersión para viscosidad cannon versus experimentos. Exponencial: $\mathrm{Y}=\exp \left(\mathrm{a}+\mathrm{b}^{*} \mathrm{X}\right)$ Coeficiente de Correlación $=0.880759$, Rcuadrada $=77.5736$ porciento, como se muestran en los gráficos 4 .
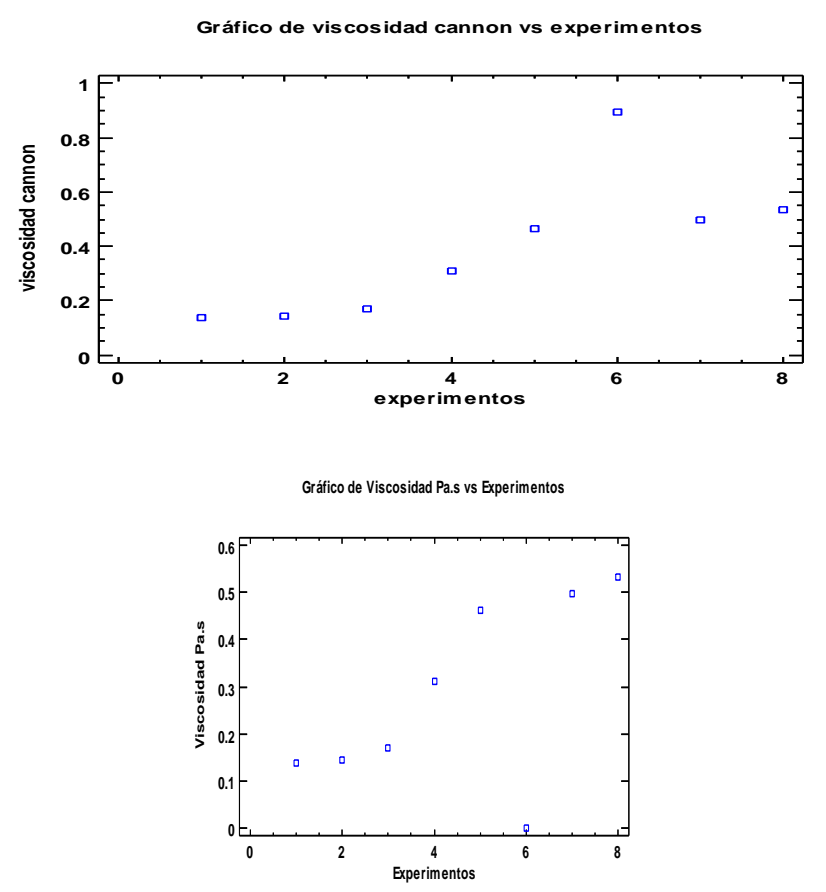

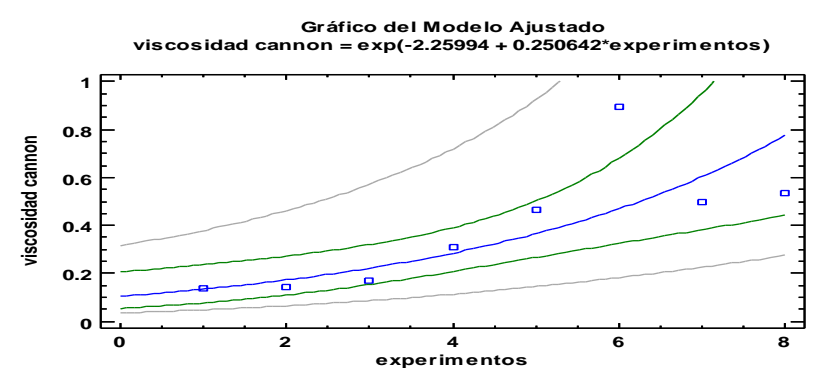

Grafico 4 Comportamiento de la viscosidad a temperatura constante de $40^{\mathrm{a}} \mathrm{C}$, viscosímetro n0 8, con un sobre otras. Volumen de $5 \mathrm{ml}$ de glicerina cruda Fuente: Elaboración Propia

Para las medidas de viscosidad se sigue el procedimiento descrito en la Norma Española UNE 14142- 55-105-73. En esta memoria nos referimos a la viscosidad cinemática, entendiéndose por viscosidad la resistencia que ofrecen las capas de una masa líquida para el desplazamiento paralelo de unas a otras. Según la Sociedad Americana de Pruebas y Materiales "ASTM" D-6751 (especificaciones en U.S.A) la viscosidad cinemática debe ser 1.9- $6.0 \mathrm{cSt}$. Se muestra en el grafico 5 la superficie de respuesta obtenida para el modelo estadístico creado para el sistema en estudio y para la variable de salida viscosidad.

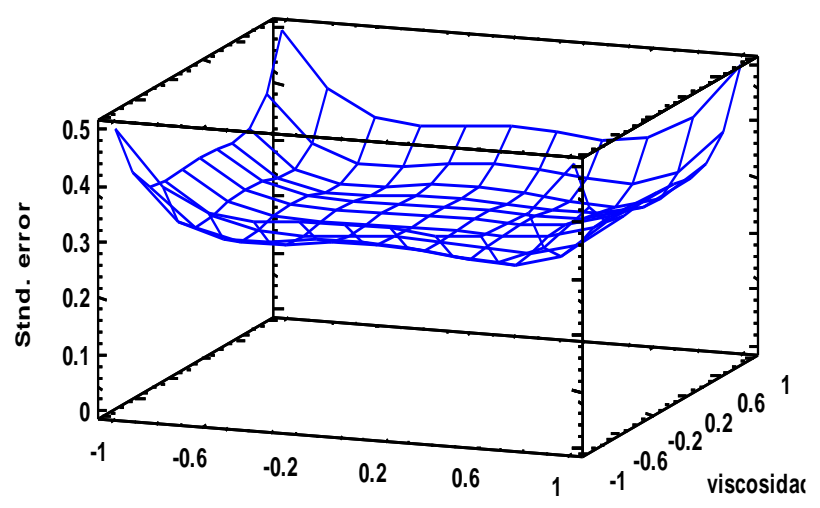

Grafico 5 Superficie de respuesta del comportamiento de la viscosidad de la glicerina cruda obtenida como subproducto del biodiesel

Fuente: Elaboración Propia

\section{Conclusiones}

En la presente investigación, para conocer y determinar las características física y químicas de la glicerina obtenida de un biocombustible, se determinaron 5 parámetros los cuales nos basamos en las normas internacionales y en ocasiones las mexicanas las NMX para grasas y aceites, glicerina para tener una mejor expectativa sobre el comportamiento de la glicerina sin tratamiento, ( cruda) ha dado lugar una revisión bibliográfica complementaria con el fin de situar esta investigación en el escenario más actual posible. 
Las muestras de glicerina de las 8 muestras, 3 se encontraban en forma semisólida las cuales por tratamiento térmico se vuelve liquida para poder determinar los parámetros de estudio, la muestra numero 7 la glicerina tenía una consistencia semisólida de color blanca de consistencia tipo cera como se indica en la siguiente figura no.11.
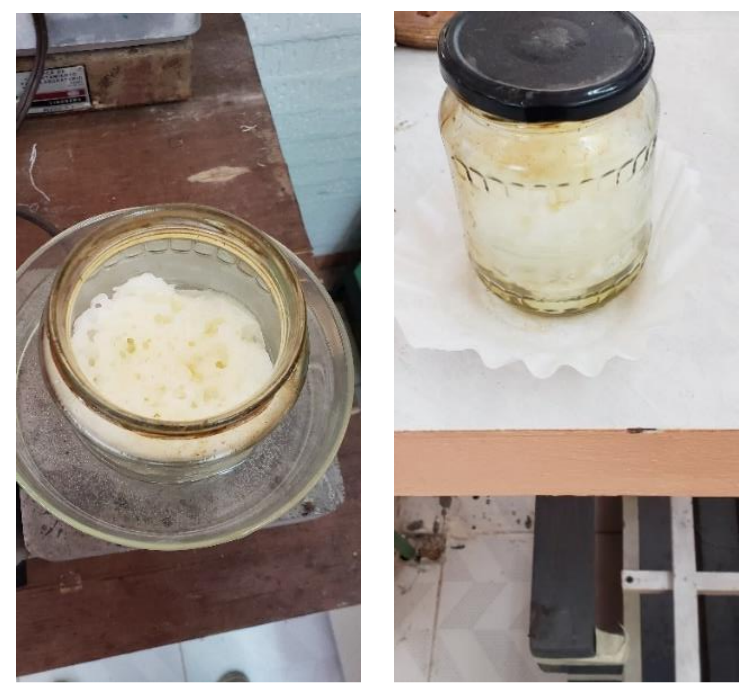

Figura 11 Muestra 7 glicerina semisólida Fuente: Elaboración Propia

El color del aceite comestible usado depende del número de frituras que ha sufrido y la humedad son alta por la ubicación geográfica donde estamos situados, por ende, es bueno tener en cuenta que a esta materia prima se le debe hacer un pre tratamiento muy riguroso para acondicionar los aceites, para obtener biodiesel y el $10 \%$ de glicerina cruda,

De los 4 cumplieron con los parámetros de acuerdo con la norma UNE-EN 14214. La recuperación del metanol solo se pudo obtener el $58 \%$ del metanol por destilación, ya que por la técnica del roto vapor no logramos obtener el máximo que indica la norma y la literatura del $98 \%$. , solo obtuvimos el $18 \%$, esto se concluye que la muestra al darle tratamiento térmico se evaporaba una parte del metanol, ya que las muestras 3, 4 se encontraban en estado sólido y semisólido.(Véase tabla 4, figura 12)

\begin{tabular}{|l|r|}
\hline Muestra & Volumen recuperado \\
\hline 1 & $58 \%$ \\
\hline 2 & $15 \%$ \\
\hline 3 & $10 \%$ \\
\hline 4 & $10 \%$ \\
\hline 5 & $20 \%$ \\
\hline 6 & $5 \%$ \\
\hline 7 & $5 \%$ \\
\hline 8 & $40 \%$ \\
\hline
\end{tabular}

Tabla 4 Recuperación del metanol

ISSN 2410-3934

ECORFAN® Todos los derechos reservados

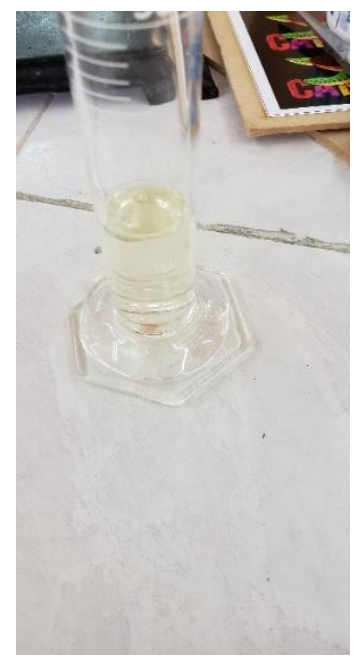

Figura 12 Cantidad colectada en el proceso recuperación del metanol por roto vapor. Y destilación Fuente: Elaboración Propia

En cuanto al $\mathrm{pH}$ se concluye que están dentro lo establecido del rango de 7 a 8 , ya que no se le aplico ningún tipo de tratamiento a dicha glicerina. Es tipo de glicerina se puede utilizar para limpieza y desengrasante de pisos, cuando se le aplica un tratamiento acido, y se refina sus aplicaciones son elaboración de velas añadiéndoles colorantes y aromas, para elaboración de jabones sólidos y líquidos. Y de esta manera tenemos un proyecto integral sustentable.

Los resultados demuestran la necesidad de generar conciencia en el uso y cuidado de nuestro ambiento, cuidando no tirando los aceites que se usan en frituras a las tarjas. Es indispensable crear conciencia sobre la disposición de los aceites usados comestibles, generando una cultura de almacenar en recipientes y después llevarlos a los centros de acopio para su tratamiento Fue quizás inesperado el haber encontrado que nuestra sociedad no tiene hábitos de reciclaje y almacenaje y disposición final del aceite usado en las cocinas y dar a conocer que ese aceite puede traer grandes beneficios ambientales $\mathrm{y}$ porque no económicos.

Impacto o beneficio en la solución a un problema relacionado con el sector productivo o la generación del conocimiento científico $\mathrm{o}$ tecnológico - - En lo científico: la generación de conocimiento científico acerca de las condiciones óptimas de producción de biodiesel y de reciclaje de aceites comestibles usados y el uso de la glicerina obtenida del biodiesel además es un tema poco conocido dentro la cultura ambiental. - 
En lo económico: el aprovechamiento de los recursos ambientales y la reducción de los impactos negativos sobre estos, reduciendo el uso excesivo y el daño a los ecosistemas, que incrementa los costos de su protección y conservación. - En lo social: la contribución para realizar concientización social acerca de los problemas relacionados con el tema de: almacenaje del aceite comestible usado, disposición del mismo en los centros de acopio.

\section{Agradecimiento}

Al Tecnológico Nacional de México /Instituto Tecnológico de Cancún, por las instalaciones de los laboratorios de química y de Biodiesel para el desarrollo del presente trabajo.

\section{Referencias}

Ávila Orozco Francisco David Biodiesel: Estudios Analíticos y Desarrollo de Métodos de Análisis para el Control de Calidad, Tesis doctoral en Química, 2015

Betancourt-Aguilar Carmen, Mello-Prado Renato, Castellanos-Gonzáles Leónides, SilvaCampo Cid N.

Características de la glicerina generada en la producción de biodiesel, aplicaciones generales y su uso en el suelo Versión On-line ISSN 18194087 cultrop vol.37 no.3 La Habana jul.set. 2016 http://dx.doi.org/10.13140/RG.2.1.4329.2403 ht tp://dx.doi.org/10.13140/RG.2.1.4329.2403

(Diario Oficial de la Federación) 2008. Ley de promoción y desarrollo de los Bioenergéticos. México, Gobierno de la República, publicada el 1 de febrero. Recuperado el 26 de noviembre de 2018. 12 p. de http://www.diputados.gob.mx/LeyesBiblio/ref/l pdb.htm.

Larosa R., Proceso para la producción de biodiesel Refinaciòn de glicerina,2009, http://www.biodiesel-

uruguay.com/articulos/Biod-rev2.pdf (revisado 2018)

Montoro Garcìa Francisco Javier 2016) Poliésteres biodegradables aprovechando el glicerol concomitante en la producción de biodiesel. Tesis de Grado, Universidad de Jaén Escuela Politécnica Superior de Linares

\section{Pacheco Román Francisco}

Reporte de Inteligencia Tecnológica Biodièsel CONACYT-Secretaría de Energía Sustentabilidad Energética (SENER) 2015 Biodiésel - Gobierno de México

Posada-duque, J. A. \& Cardona-Alzate, C. A. Análisis de la refinación de glicerina obtenida como coproducto en la producción de biodiesel. Universidad de Bogotá (Colombia), 2015. https://www.gob.mx/.../Reporte_Inteligencia_T ecnologica_BIODIESEL_270318

Torres L, Ben-Youssef. D-Ek y R-Escalante Caracterización de los parámetros fisicoquímicos de los aceites comestibles usados para la generación de biodiesel en la planta piloto del IT Cancún. Revista de Investigación y Desarrollo 2016 Vol.2 No.6 92-108 http://www.srsbiodiesel.com/technologies/glyce rin-purification/glycerin-specifications/

SRS ENGINEERING CORPORATION [web en línea]. 2008. [Consulta: 05-09-2019] 